\title{
Function and mechanism of map4k4 genes on milk traits and scs between dairy cow and buffalo (Abstract)
}

\author{
Dinesh Bhattarai, Shujun Zhang* \\ , Huazhong Agricultural University, Wuhan, China \\ *Corresponding author: sjxiaozhang@mail.hzau.edu.cn
}

\begin{abstract}
1. The variation of MAP4K4 gene has a significant effect on the number of somatic cells in milk. The objective of this study is to explore the association of single nucleotide polymorphisms present in $M A P 4 K 4$ gene with different milk traits in dairy cows. Milk production traits including milk yield, fat percentage, and protein percentage from 500 Holstein cows were collected by using 305 days lactation records. Association between MAP4K4 genotype and different traits and Somatic Cell Score (SCS) was performed using General Linear Regression Model of R. Two SNPs at exon 18 (c.2061T $>$ G and c.2196T $>$ C) with genotype TT in both SNPs were found significantly higher for somatic SCS. Thus, MAP4K4 gene could be a useful candidate gene for mastitis and the identified polymorphisms might potentially be strong genetic markers.

2. The promoter sequence of MAP4K4 gene in dairy cattle and buffalo significantly affected the expression level of MAP4K4. we identified the differences between the core promoter region of the cow and buffalo, the promoter region from -1100 to -778 bp was essential for cow-MAP4K4 while -608 to +176 bp was essential for the buffalo-MAP4K4 that the two regions contained the core functional promoter to maintain the promoter activity. The core promoter activity of cow-MAP4K4 was significantly higher $(\mathrm{P}<0.05)$ than buffalo$M A P 4 K 4$, might imply the susceptibility of mastitis and other inflammatory disease more in cow than buffalo.
\end{abstract}

Keywords: Mastitis, somatic cell score (SCS), MAP4K4, SNP, Core Promoter. 
